

\title{
AN EXACT MULTIVARIATE MODEL-BASED STRUCTURAL DECOMPOSITION
}

\author{
José Casals \\ Miguel Jerez† \\ Sonia Sotoca \\ Departamento de Fundamentos del Análisis Económico II \\ Facultad de CC. Económicas y Empresariales \\ Universidad Complutense de Madrid
}

ABSTRACT

We describe a simple procedure for decomposing a vector of time series into trend, cycle, seasonal and irregular components. Contrary to common practice, we do not assume these components to be orthogonal conditional on their past. However, the state-space representation employed assures that their smoothed estimates converge to exact values, with null variances and covariances. Among other implications, this means that the components are not revised when the sample increases. The practical application of the method is illustrated both with simulated and real data.

Keywords: State-space models, seasonal adjustment, trends, unobserved components.

JEL Classification: C32, C53, E27, E37

$$
\begin{aligned}
& 139965073 \\
& 5 \% 1942040
\end{aligned}
$$

\footnotetext{
† Corresponding author. Departamento de Fundamentos del Análisis Económico II. Universidad Complutense de Madrid. Campus de Somosaguas. 28223 Madrid (Spain). Phone: (+34) 913942361 Fax: (+34) 9139426 13. E-mail: mjerez@ccee.ucm.es.
} 


\section{Introduction.}

Many works define the additive structural decomposition of a time series, $z_{\ell}$, as

$$
z_{t}=t_{t}+c_{t}+s_{\mathrm{t}}+\varepsilon_{t}
$$

where:

ent, representing the long-term

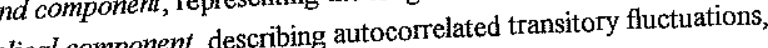

$c_{t}$ is the cyclical component, describing autocorrelated tratterns over seasons, and

$s_{t} \quad$ is the seasonal component,

$\varepsilon_{c}$ : is an irregular component.

londing interest to economists. Early

oflong-standing interest to economists. Early research emplasized the extraction of the seasonal component to obles without the "contamination" of useful in assessing the importance of fluctuations in economic variat the with component, associated with seasonal variability. In Macroeconomics, interest soon extendedto the short-term fluctuations. long-run growth, and to the transitory components, interpretable as short-term fluctuations.

(here are two basic approaches to obtain the unobserved components (UC) on the right-hand-side (1) ad-hoc methods and model-based methods.

a consist of filtering the series by means of a differential equation, designed to 列 extract the components generating peaks of spectral power at previously chosen fisin et al. (1967) and famous examples of this approximation are in the Census X-11 Findley et al (1998), An influential proposal specialized in trend thods have a clear advantage in Hodrick and Prescott (1980). The simplest versions of these methods have a clear application often simplicity, as they do not require any previous analysis. However, their mech series follow the same ields spurious decompositions, as they implicitly assume that different time s stochastic process.

Potential inadequacy of ad-hoc methods inspired the model-based methods, which emphents. This coherence between the properties of the observed series and those of the stased (AMB), the Forecas principle is at the basis of three methodologies, the ARIMA-model-based (AMR Decomposition (FD) and the Structural Time Series Models (STSM) approaches.

A AMB techniques were oignaly des (1997). A recent Tiao (1982) and Bell and Hillmer (1984). For a self-con1an) These methods start from a reducedsoftware implementation is due to Gómez and Maravall (1996), Thesestation defined by individual form ARIMA model for $z_{t}$, and afterwards obtain a structural representation defineds
ARIMA processes for each UC, constrained that their sum is observationally equivalent to the reducedform model.

The FD approach is due to Box et al. (1987), for a recent paper on FD see Espasa and Peña (1995). It consists of decomposing the $h$-steps-ahead forecast function of a given univariate model, generally belonging to the ARIMA family, into persistent and transitory components, which can also be broken down into seasonal and nonseasonal terms.

Last, STSM are directly set up in terms of the components in (1), which are represented by statespace (from now on SS) models specified according to the statistical properties of the time series, see Engle (1978), Harvey (1989), Harvey and Shephard (1993) and Young et al. (1999). Whereas AMB and FD techniques are essentially univariate, the simpler structure of SS models makes it easy to define STSM for vectors of time series and allows extensions to nonlinear and non-gaussian systems or models with stochastic variances. This approach is implemented with some differences in three main software packages: MICRO-CAPTAIN, see Young and Brenner (1991), BATS, see Pole et al. (1994), and STAMP, see and Koopman et al. (1995).

Once the models for the components have been specified and estimated using any of these methodologies, the final step in the analysis consists of estimating the components. To this purpose mos approaches use a class of algorithms known in general as "symmetric filters" such as, e.g., the WienerKolmogorov filter, see Burman (1980) and Bell and Hillmer (1984), and the fixed-interval smoother, see Anderson and Moore (1979). The word "symmetric" alludes to the fact that current estimates of the components depend on past and future values of the time series. FD methods are an important exception to this general approach, because the components implied by a forecast function depend only on past sample values and, therefore, one-side asymmetric filters are natural choices for extracting the empirical components.

The literature about structural decomposition is so rich that any generalization is deemed to be incorrect in specific cases. However, we can say that most ad-hoc, AMB and STSM methods share three main shortcomings: first, they need arbitrary restrictions; second, theoretical and empirical components have different properties; and third, estimates of the components change when the sample increases.

Arbitrary restrictions are necessary because, in general, Eq. (1) and the models for the components make up an underidentified econometric model in structural form. To improve identificability, most methods assume that the components are uncorrelated. This restriction is not justified on economic grounds but has a compelling practical motivation because structural components are often analyzed separately and, therefore, some kind of independence among them is desirable. When independence of the components is not enough to achieve exact identification, the literature suggests other restrictions such as canonical constraints, see Hillmer and Tiao (1982). 
Second, the statistical properties of theoretical and empirical components are different because, whereas the theoretical components depend only on past information, their symmetric filtered estimates depend on both, past and future information, see e.g. Planas (1997, pp. 113). As a consequence, the empirical components: a) violate the independence assumption, see García-Ferrer and del Hoyo (1992), and b) are generated by stochastic processes which do not derive from the model of the time series.

Finally, the use of symmetric filters also implies that the empirical components change when the sample increases. These "revisions" are clearly a major problem when the decomposition is applied to generate public macroeconomic information.

The empirical components obtained by FD methods avoid these general shortcomings because they are obtained from a reduced-form model, so they do not require arbitrary identification assumptions, and are extracted by filtering only past information, thus avoiding revisions and distortions in their stochastic structure. However, using only past information implies a certain inefficiency and heterogeneity of the within-the-sample structural components, as the values computed in $t$ are based in a different information set than those computed in any other instant. Also there is some ambiguity about the choice of the leadtime for the forecast function.

In this paper we use SS techniques to obtain the structural decomposition of a vector of time series generated by a linear stochastic process, addressing the shortcomings mentioned above. Section 2 defines the basic notation, summarizes some previous results about the SS representation of the data generating process and charactenizes the structural components. To do this, we state a one-to-one correspondence between the eigenvalues of the transition matrix in the SS representation and the corresponding frequencies displaying peaks of spectral power. Using consensus ideas about the properties of the components, the state variables with unit eigenvalues are associated with the trend, those with peaks at seasonal frequencies are assigned to the seasonal component and the rest of the states are included in the cycle term. The structural components are then defined by unique linear combinations of the state variables, characterized by the coefficients in the observation equation. Section 3 discusses the estimation of the structural components. As the SS representation employed is observable, the symmetric and asymmetric filtered estimates of the states (and therefore, those of the structural components) converge to conditionally orthogonal values with null variances. In this sense, the decomposition obtained is exact. Section 4 organizes the results in Sections 2 and 3 into a five-stage methodology. Practical application of our proposal is illustrated by the examples in Sections 5 and 6 . Finally, Section 7 summarizes the main conclusions and suggests some additional applications.

\section{Model representations and characterization of the structural components.}

Assume that a structural decomposition analogous to (1) is to be computed for the $m \times 1$ randon vector $z_{t}$. For reasons that will be discussed in Section 3 , our method requires $z_{t}$ to $b e$ steady-state innovations SS model (hereafter "innovations method requires $z_{f}$ to be the output of

$$
\begin{aligned}
& x_{t+1}=\Phi x_{t}+\Gamma u_{t}+E a_{t} \\
& z_{t}=H x_{t}+D u_{t}+a_{t}
\end{aligned}
$$

where:

$x_{t} \quad$ is an $n \times 1$ vector of state variables,

$u_{t}$ is an $r \times 1$ vector of exogenous variables,

$a_{t} \quad$ is an $m \times 1$ vector of errors, such that $a_{t} \sim \operatorname{iid}(0, B)$

The transition equation $(2)$ cha

observation equation (3) describes how $z_{t}$ is generated by the structure of $z_{t}$. On the other hand, the dynamic components, given by $\boldsymbol{H} \boldsymbol{x}_{t}$, b) the instanted by the surn of: a) a linear combination of the $\boldsymbol{D} \boldsymbol{u}_{t}$, and c) the error $\boldsymbol{a}_{t}$.

In Subsection 2.1 we will discuss how to: a) ob econometric model, and b) redefine the state variables intopresentation as (2)-(3) from a standard components. Subsection 2.2 discusses the spectrables into independent and clearly defined dynamic ideas, associates them to the stuct spectral properties of the state variables and, using consensus structural decomposition and relates its components. Finally, Subsection 3.3 defines a generalized innovations model.

\subsection{Previous results about the innovations model}

The representation (2)-(3) can be obtained in two ways, corresponding to Results 1.1 and 1.2 .

Result 1.1. If the model for $z_{t}$ is (or can be expressed as) a general time-invariant SS model:

$$
\begin{aligned}
& x_{t+1}^{*}=\Phi x_{t}^{*}+\Gamma u_{t}+E^{*} w_{t} \\
& z_{t}=H x_{t}^{*}+D u_{t}+C v_{t}
\end{aligned}
$$

where the errors $w_{t}, v_{t}$ are independent of the initial state $v_{t} \sim \operatorname{iid}(0, R), \operatorname{cov}\left(w_{t} v_{t}\right)=S$ for all $t=1,2, \ldots, N$ and b) $x_{1}^{*} \sim\left(\mu_{1}, \Sigma_{1}\right)$. 
Then under weak assumptions, see Casals et al. (1999, Theorem I), $z_{t}$ is also the output of the innovations model (2)-(3) with:

$$
x_{1} \sim\left(\mu_{1}, \Sigma_{1}-P\right)
$$

$$
\begin{aligned}
& P=\Phi P \Phi^{T}+E^{*} Q E^{* T}-E^{*} B E^{* T} \\
& E=\left(\Phi P H^{T}+E^{*} S C^{T}\right) B^{-1} \\
& B=H P H^{T}+C R C^{T}
\end{aligned}
$$

Note that model (4)-(5) includes two error terms, $w_{t}$ and $v_{t}$, whereas the innovations model has only one, $a_{t}$. In some sense, this means that the relationship between both formulations is analogous to that existing between the structural and the reduced-form of an econometric model, i.e., both are observationally equivalent but some information that is explicit in the covariance matrices of the "structural model" (4)-(5), $Q, R$ and $S$, is lost when they are combined in the "reduced-form model" covariance, $\boldsymbol{B}$. As we will see later, this is a very relevant fact for some applications

Result 1.1 has the advantage of generality, as it supports any model that can be written as a andard linear fixed-coefficients SS model. However, it requires to solve the nonlinear equations (7)(9). Ionescu et al (1997) describe efficient and stable procedures to do this, but this can be an undesirable complication for many users. Therefore, if the model for the time series is (or can be written as) a VARMAX process, then Result 1.2 is more convenient

Result 1.2. Assume that $\ell_{t}$ follows the VARMAX $(p, s, q)$ process

$$
F(B) z_{t}=G(B) u_{i}+B(B) a_{t}
$$

where $a_{t}$ is a $m \times 1$ vector of white noise errors, $u_{t}$ is a $r \times 1$ vector of exogenous variables; the polynomial matrices $F(B), G(B)$ and $\Xi(B)$, are defined by:

$$
\boldsymbol{F}(B)=\boldsymbol{I}+\sum_{i=1}^{p} \boldsymbol{F}_{i} B^{i}, \quad \boldsymbol{G}(B)=\sum_{i=0}^{s} \boldsymbol{G}_{i} B^{i}, \quad \Xi(B)=\boldsymbol{I}+\sum_{i=1}^{q} \Xi_{i} B^{i}
$$

and may contain roots in the unit circle; finally, $B$ denotes the backward-shift operator, such that for any sequence $x_{t}: B^{ \pm k} x_{t}=x_{t \neq k}$

Under these conditions, (10)-(11) can be expressed in the equivalent innovations form defining:
$\Phi=\left[\begin{array}{ccccc}-F_{1} & I & 0 & \ldots & 0 \\ -F_{2} & 0 & I & \ldots & 0 \\ \vdots & \vdots & \vdots & \ddots & \vdots \\ -F_{k-1} & 0 & 0 & \cdots & I \\ -F_{k} & 0 & 0 & \ldots & 0\end{array}\right], \quad \Gamma=\left[\begin{array}{c}G_{1}-F_{1} G_{0} \\ G_{2}-F_{2} G_{0} \\ \vdots \\ G_{k-1}-F_{k-1} G_{0} \\ G_{k}-F_{k} G_{0}\end{array}\right], \quad E=\left[\begin{array}{c}\Xi_{1}-F_{1} \\ \Xi_{2}-F_{2} \\ \vdots \\ \Xi_{k-1}-F_{k-1} \\ \Xi_{k}-F_{k}\end{array}\right]$

$H=\left[\begin{array}{llll}I & 0 & \cdots & 0\end{array}\right], \quad D=G_{0}$

where the state vector $\boldsymbol{x}_{\boldsymbol{t}}$ has $n=m \cdot k$ rows, and $k=\max \{p, s, q\}$, see Aoki (1990) and Terceiro $(1990)$, and the error term in the innovations model is the same as in model (10).

After obtaining a innovations model for the data, the problem consists of restructuring its dynamics in an equivalent and more meaningful representation. This can be done using Result 2

Result 2. If $z$, is realized by (2)-(3), then it is also realized by the model

$$
\begin{aligned}
& \bar{x}_{t+1}=\bar{\Phi} \bar{x}_{t}+\bar{\Gamma} u_{t}+\bar{E} a_{t} \\
& z_{t}=\bar{H} \bar{x}_{t}+D u_{t}+a_{t}
\end{aligned}
$$

where the states in (14)-(15) are related to those in (2)-(3) by a linear transformation $\overline{\boldsymbol{x}}_{\boldsymbol{t}}=\boldsymbol{U} \boldsymbol{x}_{t}$, such that $|U| \neq 0$. Accordingly, the matrices in (14)-(15) are related to those in (2)-(3) by the expressions: $\bar{\Phi}=U \Phi U^{-1}, \bar{\Gamma}=U \Gamma, \bar{E}=U E, \bar{H}=\boldsymbol{H} U^{-1}$, and the transformed transition matrix $\bar{\Phi}$ is blockdiagonal. The dimensions of these blocks can be: $1 \times 1$, representing a unique real eigenvalue, $2 \times 2$, representing a pair of imaginary eigenvalues, and $v \times v$, representing $v$ repeated eigenvalues.

This factorization of a transition matrix can be obtained by different procedures, being the Jordan decomposition the most popular algorithm. For the purposes of this paper, we use a simpler and more stable method, which consists of applying the Schur decomposition to $\Phi$ and diagonalizing the resulting real Schur matrix by solving a system of Sylvester equations. For a complete description of this procedure, see Petkov et al (1991, pp. 103-106).

\subsection{Frequency-domain properties of the state variables.}

The block-diagonal representation (14)-(15) decomposes the dynamic response of the system to shocks in the inputs, $\boldsymbol{\alpha}_{\boldsymbol{t}}$ and $\boldsymbol{u}_{\boldsymbol{t}}$, into several basic movements corresponding to different eigenvalues of the transition matrix. These basic movements, which are termed latent components by West (1997), represent independent reactions of the state variables.

In the domain of time it is difficult to associate these latent movements with the structural components. In the frequency domain, however, all the structural components can be clearly defined. 
Following Burman (1980), the trend is represented by a peak at low frequencies, the irregular component should display no significant peak at any frequency, the seasonal component comprises the spectral peaks at the basic seasonal frequency and its multiples. By exclusion, any other component

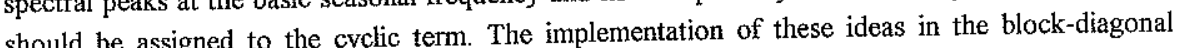
innovations model (14)-(15) is easy, as the eigenvalues of the transition matrix characterize unambiguously the properties of the different states in both time and frequency domains.

Assume that $\lambda_{j, k}=a_{j} \pm b_{j} i$ is a pair of conjugate eigenvalues of $\bar{\Phi}$, associated with the states $j$ and $k$. Under these conditions, it is easy to show that both states generate a peaks in the pseudo-spectrum in the frequency $f_{j, k}=(2 \pi)^{-1} \arctan \left(b_{j} / a_{j}\right)$, where $f_{j, k}$ is in cycles per unit time. There are several particular cases worth considering:

1) If the real part of $\lambda_{j, k}$ is zero, then $b_{j} / a_{j}=\infty$, implying that $f_{j, k}=1 / 4$ if $b_{j}>0$ or $f_{j, k}=3 / 4$ if $b_{j}<0$.

2) If an eigenvalue is a real number, then $b_{j} / a_{j}=0$, implying that $f_{j, k}=0$ if $a_{j}>0$ or $f_{j, k}=1 / 2$ if $a_{j}<0$.

3) If $b_{j}=0$ and $a_{j}$ tends to one, the spectral power tends to infinity at $f_{j, k}=0$

According to these results, and assuming that the system is not explosive, the states in the blockdiagonal model can be naturatly assigned to the structural components with the rules summarized in Table 1

\section{[Insert Table 1]}

where $F_{\text {}}$ is the set of seasonal frequencies defined as $\boldsymbol{F}_{s}=\left\{f_{j}=k_{j} / s ; k_{j}=1,2, \ldots,[s / 2]\right\}$, being $s$ the seasonal period and $[s / 2]=s / 2$ if $s$ is even, or $[s / 2]=(s-1) / 2$ if $s$ is odd.

Therefore, all the states with a unit eigenvalue are assigned to the trend component, all the states generating peaks at nonseasonal frequencies are assigned to the cycle component, and all the states (the seasonal component. Note that in a SS

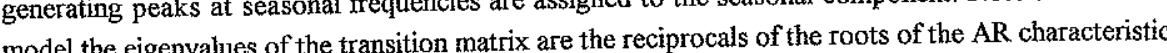
model the eigenvalues of the transition matrix are the reciprocals of the roots of the AR. characteristic
polynomial. Then, an assignation analogous to that in Table 1 can be made in terms of the AR roots.

\subsection{Characterization of the structural components.}

Assume that $z_{t}$ is generated by the block-diagonal innovations model (14)-(15) Consider also the multivariate extension of (1):

$$
z_{t}=t_{t}+c_{t}+s_{t}+d_{t}+\varepsilon_{t}
$$

where $t_{t}, c_{t}, s_{t}$ and $\varepsilon_{t}$ are $m \times 1$ vectors of trend, cycle, seasonal and irregular components, respectively. A new term, $d_{t}$, represents the instantaneous effects of exogenous variables on $z_{t}$. In a framework of structural decomposition, this term is often used to model calendar effects or outliers.

Under such conditions, the components in (16) can be characterized by restructuring the blockdiagonal model as:

$$
\begin{aligned}
& {\left[\begin{array}{l}
\bar{x}_{t+1}^{t} \\
\bar{x}_{t+1}^{c} \\
\bar{x}_{t+1}^{s}
\end{array}\right]=\left[\begin{array}{ccc}
\bar{\Phi}^{t} & 0 & 0 \\
0 & \bar{\Phi}^{c} & 0 \\
0 & 0 & \bar{\Phi}^{s}
\end{array}\right]\left[\begin{array}{l}
\bar{x}_{t}^{t} \\
\bar{x}_{t}^{c} \\
\bar{x}_{t}^{s}
\end{array}\right]+\left[\begin{array}{l}
\bar{\Gamma}^{t} \\
\bar{\Gamma}^{c} \\
\bar{\Gamma}^{s}
\end{array}\right] u_{t}+\left[\begin{array}{l}
\bar{E}^{t} \\
\bar{E}^{c} \\
\bar{E}^{s}
\end{array}\right] a_{t}} \\
& z_{t}=\left[\begin{array}{lll}
\bar{H}^{t} & \bar{H}^{c} & \bar{H}^{s}
\end{array}\right]\left[\begin{array}{c}
\bar{x}_{t}^{t} \\
\bar{x}_{t}^{c} \\
\bar{x}_{t}^{s}
\end{array}\right]+D u_{t}+a_{t}
\end{aligned}
$$

where $\bar{x}_{t}^{t}$ is the vector of nonstationary states, $\bar{x}_{t}^{c}$ is the vector of stationary (nonseasonal) states and $\bar{x}_{t}^{s}$ is the vector of seasonal states. Accordingly, the structural components are defined as:

$$
\begin{aligned}
& t_{t}=\bar{H}^{t} \bar{x}_{t}^{t} \\
& c_{t}=\bar{H}^{c} \bar{x}_{t}^{c} \\
& s_{t}=\bar{H}^{s} \bar{x}_{t}^{s} \\
& d_{t}=D u_{t} \\
& \varepsilon_{t}=a_{t}
\end{aligned}
$$

Note that there are infinite block-diagonalizing matrices, $U$, but the components (19)-(23) are unique, because block-diagonalization is a similar transformation of the original transition matrix.

Expressions (16) and (19)-(23) can be interpreted as a decomposition of the one-step-ahead forecast function. To see this, note that (23) defines the irregular component to be the error term of the econometric model. Then the within-the-sample components in (19)-(22) add up to the corresponding fitted value, whereas the component in (24) coincides with the residual. When computed out of the sample, (19) (22) add up to the corresponding forecast. 


\section{Estimation of the structural components.}

Given the characterization of the structural components made in Section 2, the problem reduces to obtaining estimates of the state variables and combining them according to (19)-(23). The SS literature provides two basic algorithms to do this: the Kalman filter and the fixed-interval smoother literature provides two basic algorithms to do this. the Kalman While the former is a one-sided asymmetric filter, providing efficient estimates of the first and secondorder moments of the states conditional on past information, the latter is a two-sided symmetric filter order moments of the states con the sample. Therefore, FIS estimates are more precise in general. For the purposes of this paper, we use an efficient implementation of the FIS algorithm which allows for both, stationary and nonstationary roots, see Casals et al. (2000).

Denoting the information set up to time $i$ as $\Omega^{i}=\left\{z_{1}, z_{2}, \ldots, z_{i}, u_{1}, u_{2}, \ldots, u_{i}\right\}$, and the FIS estimates by $x_{t \mid N}=E\left(x_{t} \mid \Omega^{N}\right)$ and $\boldsymbol{P}_{t \mid N}=E\left[\left(x_{t}-x_{t \mid N}\right)\left(x_{t}-x_{t \mid N}\right)^{T} \mid \Omega^{N}\right], N$ being the sample size, the following result holds:

Result 3. In an innovations model, the FIS covariance, $P_{t \mid N}$ converges to zero as $t$ increases

This important property can be derived as a Corollary of Theorem 4.2. in De Souza et. al (1986) taking into account that: a) an innovations model is detectable and b) the strong solution of the corresponding Riccati equation is zero. For our purposes, the following argument is simpler and more direct.

Proof. Eq. (3) implies that $\boldsymbol{a}_{\boldsymbol{t}}=\boldsymbol{z}_{\boldsymbol{t}}-\boldsymbol{H} \boldsymbol{x}_{\boldsymbol{t}}-\boldsymbol{D} \boldsymbol{u}_{\boldsymbol{t}}$. Substituting this expression in (2) yields:

$$
x_{t+1}=(\Phi-E H) x_{t}+(\Gamma-E D) u_{t}+E z_{t}
$$

If the initial states $x_{1}$ were known, all the sequence of FIS states would be exactly determined by (24) because the inputs in the right-hand-side of this expression are contained in $\Omega^{N}$. In most cases $x_{1}$ is unknown and treated as a random variable. Then the covariance of smoothed estimates, conditional on all the sample, would be:

$$
P_{t \mid N}=(\Phi-E H)^{t-1} P_{1 \mid N}\left[(\Phi-E H)^{t-1}\right]^{T}
$$

and the eigenvalues of $\Phi-E H$ coincide with the reciprocal roots of the MA terms, see e.g. (11) and (12)-(13). Then if the model is invertible, i.e., if the eigenvalues of $\Phi-\boldsymbol{E H}$ are inside the unit circle, $\boldsymbol{P}_{\boldsymbol{t} \mid N}$ (12)-(13). The if he converges to zero at an exponential rate as $t$ increases. If the MA polynomial has roots on the unit circle the convergence of $\operatorname{tr}\left(\boldsymbol{P}_{t \mid N}\right)$ to zero is more complex.

Result 3 implies that the states in an innovations model are observable, i.e, its FIS estimates converge to exact values. Therefore, the FIS states and the empirical structural components, which are linear combinations of these states, can be treated as actual data and interpreted separately, as they are mutually independent. Also Result 3 assures that, after convergence to null covariances has been achieved, the empirical structural components will not be revised, as FIS revisions are proportional to the uncertainty affecting the estimates, see Casals et al. (2000).

The following example illustrates the different modes of convergence implied by Result 3

Example. Consider the models:

$$
\begin{aligned}
& \left(1-1.5 B+.5 B^{2}\right) z_{t}=a_{t}, \sigma_{a}^{2}=.1 \\
& \left(1-1.5 B+.5 B^{2}\right) z_{t}=(1-.8 B) a_{t}, \sigma_{a}^{2}=.1
\end{aligned}
$$

Figure 1.a. shows the trace of FIS covariance of the states in the SS representation of (26)-(27), computed with 100 simulated observations. Note that:

1) When the model has a pure autoregressive structure, as in (26), the trace becomes zero after processing a number of observations equal to the order of the process.

2) If the model has invertible moving average terms, as in (27), $\operatorname{tr}\left(\boldsymbol{P}_{t \mid N}\right)$ converges exponentially to zero as $t \rightarrow N$. The rate of convergence is governed by the decay of the coefficients in the corresponding autoregressive representation. Then, if the moving average factor is close to noninvertibility, convergence will be slower, and vice versa.

The fact that models (26) and (27) have an AR unit root does not affect the result, but unit roots in the MA factors imply a weaker convergence property. For example, if the model were:

$$
\left(1+.3 B-.5 B^{2}\right) z_{t}=(1-B) a_{t}, \sigma_{a}^{2}=.1
$$

the trace of the FIS covariance would be as shown in Figure 1.b, for 100 and 200 observations. Then when the model is noninvertible, the trace of $\boldsymbol{P}_{t \mid N}$ converges to a small value for each sample size, and this value tends to zero when the sample increases. Then, convergence of $\operatorname{tr}\left(\boldsymbol{P}_{t \mid N}\right)$ to zero is assured when $t \rightarrow \infty$.

$$
\text { [Insert Figure 1] }
$$

The following corollaries provide further insight into the implications of Result 3. 
Corollary 3.1. The Kalman filter covariance of an innovations model, $P_{t \mid t-1}$, converges to zero.

Proof The proof is identical to that of Result 3, replacing the subindex $N$ in (25) by $t-1$.

Corollary 3.2. In an innovations model the FIS and Kalman filter estimates of the states converge the same values, so that $\left\|x_{t \mid N}-x_{t \mid t-1}\right\| \rightarrow 0$, being \|\| a vector norm. $t \rightarrow \infty$

Proof. Immediate from Result 3 and Corollary 3.1

Therefore, the one-sided Kalman filter estimates also converge to exact values (Corollary 3.1) and (Corollary 3.2). These properties allow for 作 computationally efficient implementation

\section{Structure of the method.}

Building on the ideas discussed in Sections 2 and 3 , the structural decomposition of a vector of time series, $z_{t}$, can be organized in the following steps

Step 1) Obtain an adequate representation for $z_{t}$ and the equivalent innovations model, using Results 1.a or 1.b.

Step 2) According to Result 2, obtain the equivalent block-diagonal innovations model

Step 3) Compute FIS estimates of the states, $\overrightarrow{\boldsymbol{x}}_{t \mid N^{2}}$ and the corresponding covariances, $\boldsymbol{P}_{t \mid N^{N}}$, which by Result 3 converge to zero.

Step 4) Classify the different states according to their eigenvalues and compute estimates of the trend, cycle and seasonal components by combining the FIS estimates of the states with the corresponding coefficients in the observation equation, see (19)-(21).

Step 5) Compute the instantaneous effect of the exogenous variables as indicated in (22).

Step 6) Compute FIS estimates of the irregular component as $\boldsymbol{a}_{t \mid N}=\boldsymbol{z}_{t}-\overline{\boldsymbol{H}} \overline{\boldsymbol{x}}_{t \mid N}-D \boldsymbol{u}_{t}$, see (15) and (23).

To illustrate the practical application of this methodology, Sections 5 and 6 present two examples.

The first example consists of a worst-case comparison of our method with STSM and AMB decompositions, using simulated data. To this end, we simulate an stochastic process which can be interpreted both, as a STSM and an AMB decomposition. The components are then extracted using the direct SS representation and the equivalent innovations model and compared in different aspects. In this comparison, our method is in deliberate disadvantage, as the data generating process makes explicit certain variance proportionality constraints which get lost in the innovations representation. Despite this fact, the components obtained by both methodologies result very similar overall and our components have some advantages derived from their convergence to exact values. Also, it is shown that the informational disadvantage can be compensated by means of an ex-post smoothing of some components.

In the second example we illustrate the flexibility and multivariate capacities of our method by modeling two real time series. The empirical analysis suggests the existence of a common trend and common harmonic cycle. In our framework, the extraction and separate analysis of the common and specific components in both series results straightforward. Also, the presence of some missing data in the sample results only a minor inconvenient. 
5. An example with simulated data: comparison between the components implied by a structural time series model and the corresponding innovations model.

Consider the data generating process defined in Table 2:

\section{[Insert Table 2]}

Following the terminology of Harvey (1989), the trend component follows a particular case of the stochastic trend model, implying that the trend in $t+1$ is equal to the trend in $t$ plus a random walk derivative of the trend, $\Delta_{i}$. The seasonal component follows a quarterly dummy variable seasonality model, where the sum of the seasonal components over a year is a random disturbance. The error terms $\eta_{\mathfrak{t}}, \omega_{z}$ and $e_{t}$ are gaussian white noise processes, with an instantaneous covariance matrix:

$$
\operatorname{cov}\left[\begin{array}{l}
\eta_{t} \\
\omega_{t} \\
e_{t}
\end{array}\right]=\left[\begin{array}{ccc}
1 / 1600 & 0 & 0 \\
0 & .1 & 0 \\
0 & 0 & 1
\end{array}\right]
$$

note that the noise-variance-ratio, $\sigma_{\eta}^{2} / \sigma_{e}^{2}=1 / 1600$, coincides with the value assumed in the HP filter for quarterly data.

In the second column of Table 2 the model is interpreted as an AMB decomposition, where the observed time series follows an ARIMA $(0,1,5) \times(0,1,0)_{4}$ model (very close to a standard airline model), the trend follows an integrated random-walk and the seasonal component follows an AR(3) process, with all its roots on the unit circle

Table 3 shows the SS representation of the data generating process. Note that this model is not in innovations form as the shocks affecting the state variables are different from the observation error

\section{[Insert Table 3]}

Using Result 1.1, this model can be written in an equivalent innovations form, and then blockdiagonalized using Result 2 . The representation obtained in this way is shown in Table 4

\section{[Insert Table 4]}

Note that: a) the states are now clearly separated in different $(1 \times 1)$ and $(2 \times 2)$ blocks, and $b)$ the eigenvalues of the transition matrix are identical to those in Table 3. According to their frequencies, the first and second states correspond to the trend and the states third to fifth correspond to the seasonal component. Taking into account this classification of the states and the coefficients in the last row of Table 4, the structural components are given by:

$$
\begin{aligned}
& t_{t}=\bar{x}_{1 t} \\
& s_{t}=.619 \bar{x}_{3 t}-.342 \bar{x}_{4 t}-.577 \bar{x}_{5 t} \\
& \varepsilon_{t}=z_{t}-t_{t}-s_{t}
\end{aligned}
$$

Combining the SS model in Table 4 with (30)-(32), we can obtain the ARIMA models for these components. Table 5 compares them with the corresponding ARIMA representations of the data generating process.

\section{[Insert Table 5]}

Note that both sets of models have the same autoregressive structures, but different error terms. While the components of the data generating process follow pure autoregressive processes, the components implied by the innovations model receive shocks from the delayed irregular component, affected by different moving average polynomials and scale parameters.

\subsection{Comparison between the properties of the empirical components.}

We now generate 200 random draws from the data generating process, see Appendix A, and compute the FIS estimates of the components using both, the direct SS formulation in Table 3 and the equivalent innovations model in Table 4. Figure 2 shows that the trend and seasonal components obtained in both cases are very similar. The irregular components have different volatilities as expected, see Table 5 .

\section{[Insert Figure 2]}

Despite their overall similarity, the statistical properties of these components are very different. Figure 3 compares the FIS variances of the trend and seasonal components. Note that the variances of the STSM components display the "U-shape" characteristic of symmetric filters, meaning that the estimates at the center of the sample are more precise than those at the extremes. When the decomposition is applied to obtain seasonally adjusted data this fact is very important, because an increase in the sample generates a revision effect proportional to the uncertainty of the components. On the other hand, the components implied by the innovations model converge to deterministic values within the sample, as was stated in Result 3 . Therefore, its most recent values are not affected by future values of the time series. 


\section{[Insert Figure 3]}

Figure 4 shows the sample autocorrelation functions of the second-order differences of the FIS trends. Note that the autocorrelation function corresponding to the STSM trend is not white noise, despite the theoretical model for this component, see Table 5 . In comparison, the autocorrelations of the trend implied by the innovations model are coherent with its $\operatorname{ARIMA}(0,2,1)$ model

\section{[Insert Figure 4]}

The second difference of the empirical STSM trend displays a (perhaps nonstantionary) secondorder autoregressive structure. Therefore, the level of this variable has at least a fourth-order AR structure, with two or even three roots on the unit circle. This fact suggests some mutual contamination between the trend and quarterly components. As a matter of fact this was to be expected because, despite the independence constraint in (29), the FIS estimates of the components in the STSM are correlations between the trend and seasonal states in the STSM, computed from the FIS covariance matrices, vary between \pm .20 at the beginning and at the end STSM, computed from the FIS covariance matrices, vary between \pm .20 at the beginning and at the end of the those of the theoretical models.

\subsection{Comparison between the trend derivatives.}

Structural decompositions are often used to measure the turning points in the trend, see e.g. ( Garcia-Ferrer and Queralt (1998). Figure 5.a compas the derives of the STSM model and the innovations model. The latter is more volatile because the "smoothness" of trend results from the ratios between the variances in (29), which are explicit in the data generating process but not in the innovations model. Therefore, the trend derivative obtained directly from the innovations model is useless for this purpose.

If smoothness of the trend is relevant for the analysis, it can be achieved by applying the HP filte to the seasonally adjusted data obtained from the innovations model. In this case this is coherent with the theoretical model, because the variances in (29) were chosen according to the assumptions of this filter. Figure $5 . \mathrm{b}$ shows that the derivatives of the trend resulting from the STSM and the ex-pos smoothed trend are practically undistinguishable. In fact, the absolute differences between both derivatives range between $10^{-1}$ and $10^{-4}$

[Insert Figures 5.a and 5.b]

\section{An example with real data: common features in wheat prices.}

The degree of market integration in different periods has always attracted the interest of quantitative economic historians. Many of their works share two common features: a) they focus in grain markets, due to their importance in the pre-industrial economies, and b) are based in the statistical analysis of time series of prices for different grains, measured in several locations.

In this example we analyze the yearly series of prices of the wheat sold in the Monasterio de Sandoval $\left(S_{f}\right)$ and in the Fábrica de la iglesia de Alaraz $\left(A_{t}\right)$, between 1691 and $1788(N=98)$, in reales per fanega. The second series has six missing values due to discontinuities in the source, see Appendix $B$. The goals of the analysis are: a) testing if the prices in these locations display common statistical features, see Engle and Kozicki (1993) and, if found, b) apply our method to estimate them.

After a standard analysis, the following univariate models were fitted to the log-transformed series and to the difference of $(\log )$ prices:

$$
\begin{aligned}
& \left(1-.049 B+.289 B^{2}\right) \nabla \log S_{t}=\hat{a}_{t}^{S} ; \hat{\sigma}_{S}^{2}=.077 ; Q(10)=5.55 ; p=4.12, d=.54 \\
& \quad(.098)(.098) \\
& \left(1-.004 B+.343 B^{2}\right) \nabla \log A_{t}=\hat{a}_{t}^{A} ; \hat{\sigma}_{A}^{2}=.113 ; Q(10)=12.25 ; p=3.99, d^{2}=.59 \\
& \quad(.100)(.100) \\
& (1-.280 B)\left(\log A_{t}-\log S_{t}\right)=.107+\hat{a}_{t} ; \hat{\sigma}^{2}=.067 ; Q(10)=6.81 \\
& \quad(.102) \quad(.031)
\end{aligned}
$$

where the figures in parentheses are the standard deviations of the estimates; $Q(10)$ is the Ljung-Box $Q$ statistic, computed with the first 10 lags of the sample autocorrelation function; and the values $p, d$ are, respectively, the period and damping factor of the pseudo-cycle implied by the corresponding $\operatorname{AR}(2)$ factor. Due to the presence of missing values, the estimates in (34) and (35) were obtained using SS techniques, see Kohn and Ansley (1986) and Terceiro (1990, Chapter 5).

Then, single $\log$ prices are adequately represented by $\operatorname{ARIMA}(2,1,0)$ processes, implying that the series are nonstationary and have harmonic pseudo-cycles with a period of roughly four years. Also, the model for the difference of $\log$ prices is a stationary AR(1) with a constant term, meaning that both series share a common trend and a common harmonic cycle. The presence of these cofeatures supports the idea that grain markets were substantially integrated in this historical period.

Further evidence can be found by building a multivariate model for the series, constrained to represent these cofeatures. A bivariate analysis according to the methodology of Jenkins and Alavi (1981) provided the following model: 


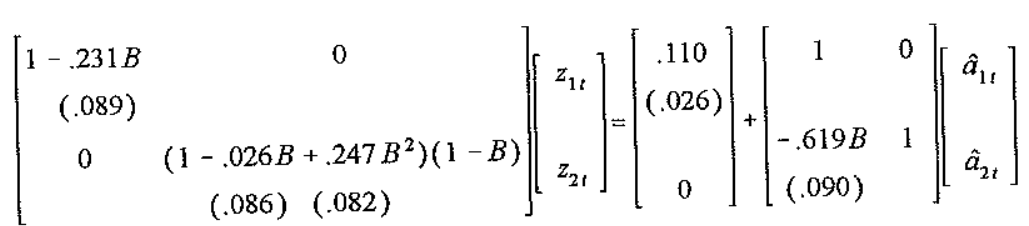

$$
\hat{\Sigma}_{a}=\left[\begin{array}{cc}
.068 & -.003 \\
= & .052
\end{array}\right] ; \quad Q(10)=\left[\begin{array}{cc}
6.99 & 11.06 \\
9.18 & 9.93
\end{array}\right]
$$

where $z_{t}$ is defined as

$$
\left[\begin{array}{c}
z_{1 t} \\
z_{2 t}
\end{array}\right]=\left[\begin{array}{cc}
1 & -1 \\
0 & 1
\end{array}\right]\left[\begin{array}{l}
\log A_{t} \\
\log S_{t}
\end{array}\right]
$$

and $Q(10)$ is a matrix of Ljung-Box statistics for the residual sample autocorrelations and crosscorrelations. Table 6 summarizes the structure of model (36)-(37) in block-diagonal innovations form. According to the eigenvalues of the transition matrix, the first state correspond to the trend, the second state corresponds to a cyclical movement and the third and fourth states correspond to a harmonic pseudo-cycle.

\section{[Insert Table 6]}

The next step consists of obtaining the FIS estimates for the states. Figure 6 represents the trace of their FIS covariance matrices. Note that the trace drops quickly to zero, with transitory peaks of uncertainty due to the effect of the missing observations.

\section{[Insert Figure 6]}

Obtaining the structural decomposition of $z_{t}$ is now trivial, taking into account the observation equation in Table 6. However, the FIS results can be combined in a more expressive form. By (38) the variables in $z_{t}$ are related with the $\log$-prices by:

$$
\left[\begin{array}{l}
\log A_{t} \\
\log S_{t}
\end{array}\right]=\left[\begin{array}{cc}
1 & -1 \\
0 & 1
\end{array}\right]^{-1}\left[\begin{array}{l}
z_{1 t} \\
z_{2 t}
\end{array}\right]
$$

therefore, pre-multiplying the observation equation by:

$$
\left[\begin{array}{cc}
1 & -1 \\
0 & 1
\end{array}\right]^{-1}=\left[\begin{array}{ll}
1 & 1 \\
0 & 1
\end{array}\right]
$$

we obtain the following decomposition of the log prices:

$$
\left[\begin{array}{l}
\log A_{t} \\
\log S_{t}
\end{array}\right]=\left[\begin{array}{llll}
.971 & 1 & 1.106 & -.391 \\
.971 & 0 & 1.106 & -.391
\end{array}\right]\left[\begin{array}{l}
\bar{x}_{1 t} \\
\bar{x}_{2 t} \\
\bar{x}_{3 t} \\
\bar{x}_{4 t}
\end{array}\right]+\left[\begin{array}{c}
110 \\
0
\end{array}\right]+\left[\begin{array}{cc}
1 & 1 \\
0 & 1
\end{array}\right]\left[\begin{array}{l}
\hat{a}_{1 t} \\
\hat{a}_{2 t}
\end{array}\right]
$$

which yields the structural components:

$$
\begin{aligned}
& t_{t}=.971 \bar{x}_{1 t} \\
& c_{t}=1.106 \bar{x}_{3 t}-.391 \bar{x}_{4 t} \\
& c_{t}^{A}=\bar{x}_{2 t}+.110 \\
& \varepsilon_{t}^{A}=\hat{a}_{1 t}+\hat{a}_{2 t} \\
& \varepsilon_{t}^{S}=\hat{a}_{2 t}
\end{aligned}
$$

According to (41), $t_{t}$ is the common trend, $c_{t}$ is the common cycle and $c_{t}^{A}$ is a stationary component including all the individual features of the log price in Alaraz. A strict application of the decomposition defined in Section 2 would imply computing a separate component for the constant term, $d_{t}=110$. Obviously this would be a meaningless complication, so we decided to add a constant to the cyclical component in (44). Last, the white noise terms, $\varepsilon_{t}^{A}$ and $\varepsilon_{t}^{S}$ are, respectively, the irregular components affecting the series of Alaraz and Sandoval, respectively. The FIS estimates of all these components are shown in Figures 7 and 8.

\section{[Insert Figures 7 and 8]}

Finally, according to (45)-(46), the covariance matrix of the irregular components is:

$$
\operatorname{cov}\left(\begin{array}{c}
\varepsilon_{t}^{A} \\
\varepsilon_{t}^{S}
\end{array}\right)=\left[\begin{array}{ll}
.114 & .049 \\
.049 & .052
\end{array}\right]
$$




\section{be a substantial degree of market integration}

After decomposing the series, the exercise could continue by looking for exogenous variables causing the cofeatures. For example, a climatological indicator could explain part of the common trend and/or the common cycle.

\section{Concluding remarks.}

The structural decomposition proposed in this paper improves existing methods in several aspects.

First, it enforces consistency between the properties of a time series and those of the structural components, as model-based methods do, but avoids the ad-hoc identification constraints required by the AMB or the STSM approaches, as it only requires a reduced-form econometric model for the data.

Second, the signal extraction algorithm employed is a symmetric FIS. Therefore, the past and future sample information is efficiently processed when computing within-the-sample empirical components.

Third, the method guarantees convergence of the empirical components to certain values, assuring coherence between the properties of the theoretical and empirical components, providing a rigorous statistical foundation for using the empirical components as observed time series and avoiding revisions of the empirical components when the sample increases. Obviously, these properties are in practice conditional on the model for the observed time series. Changes in the formulation of the model or in its parameters affect both the theoretical and empirical components.

Fourth, it easily accommodates multivariate time series and constraints upon the structural components, such as cointegration or other cofeatures, see the example in Section 6.

Last, our method is independent of particular model-building techniques or model specifications. The only requirement is that the model for the data should have an equivalent linear and time-invariant SS representation. Therefore it can be applied to many common stochastic processes such as ARIMA, VARMAX, univariate transfer functions or periodic VARMAX, using Result 1.2, and also to STSM or models with errors-in-variables, using Result 1.1. The example in Section 5 illustrates clearly this point, as the data generating process is an STSM, which has equivalent innovations and ARMA representations.

In applications not reported here, we found our decomposition to be useful in testing for common dynamic components between seasonal time series and forecasting a time series with long-run constraints on its components.

\section{Acknowledgments.}

We thank Enrique Llopis for providing the time series listed in Appendix B. Arthur B. Treadway, Jaime Terceiro, José Luis Gallego and Marcos Bujosa read early drafts of this paper and made many useful suggestions. All errors are our responsibility. 


\section{References.}

Anderson, B.D.O. and J.B. Moore (1979). Optimal Filtering, Prentice-Hall, Englewood Cliffs, N.J.

Aoki, M. (1990). State Space Modeling of Time Series, Springer-Verlag, Heidelberg,

Bell, W.R (1984). "Signal Extraction for Non-Stationary Time Series," Annals of Statistics, 12, 646664 .

Bell, W.R. and S.C. Hillmer (1984). "Issues Involved with the Seasonal Adjustment of Economic Time Series," Journal of Business and Economic Statistics, 2, 291-320.

Box, G.E.P., S.C. Hillmer and G.C. Tiao (1978). "Analysis and Modeling of Seasonal Time Series," in A. Zellner (ed.), Seasonal Analysis of Time Series, Bureau of the Census (Washington, D.C.).

Box, G.E.P., D.A. Pierce and P. Newbold (1987). "Estimating Trend and Growth Rates Analysis in Seasonal Time Series," Journal of the American Statistical Association, 82, 397, 276-282.

Burman, J.P. (1980), "Seasonal Adjustment by Signal Extraction," Journal of the Royal Statistical Society, series A, 143, 321-337.

Casals, J., Sotoca, S. y Jerez, M. (1999). "A Fast and Stable Method to Compute the Likelihood of Time Invariant State-Space Models," Economics Letters, 65, 329-337.

Casals, J., M. Jerez and S. Sotoca (2000). "Exact Smoothing for Stationary and Nonstationary Time Series," International Journal of Forecasting, 16, 1, 59-69.

De Souza, C.E., M.R. Gevers and G.C. Goodwin (1986). "Riccati Equations in Optimal Filtering of Nonstabilizable Systems having Singular State Transition Matrices," IEEE Transactions on Automatic Control, Vol. AC-31, 9, 831-838.

Engle, R.F. (1978). "Estimating Structural Models of Seasonality," in A. Zellner (ed.), Seasonal Analysis of Time Series, Bureau of the Census (Washington, D.C.)

Engle, R.F. and S. Kozicki (1993). "Testing for Common Features," Journal of Business and Economic Statistics, 11, 369-380.

Espasa, A. and D. Peña (1995). "The Decomposition of Forecast in Seasonal ARIMA Models," Journal of Forecasting, 14, 7, 565-583.

Findley, D.B., B.C. Monsell, W.R. Bell, M.C. Otto and B. Chen (1998). "New Capabilities and Methods of the X-12-ARIMA Seasonal-Adjustment Program," Journal of Business and Economic Statistics, 16, 127-177.
García-Ferrer, A. and J. del Hoyo (1992). "On Trend Extraction Models: Interpretation, Empirical evidence and Forecasting Performance," Journal of Forecasting, 11, 8, 645-665.

García-Ferrer, A. and R. Queralt (1998). "Can Univariate Models Forecast Turning Points in Seasonal Economic Time Series?”, International Journal of Forecasting, 14, 433-446.

Gómez, V. and A Maravall (1996). "Programs TRAMO and SEATS: Instructions for the User," Working paper 9628, Bank of Spain, Madrid (http://www.bde.es/servicio/software/software.htm).

Harvey, A.C. (1989). Forecasting, Structural Time Series Models and the Kalman Filter, Cambridge University Press (Cambridge).

Harvey, A.C. and N. Shephard (1993). "Structural Time Series Models," in G.S. Maddala, C.R. Rao and H.D. Vinod (eds.), Handbook of Statistics, vol. 11, 261-302, Elsevier Science Publishers, Amsterdam.

Hillmer, S.C. and G.C. Tiao (1982). "An ARIMA-Model-Based Approach to Seasonal Adjustment," Journal of the American Statistical Association, 77, 63-70.

Hodrick, R.J. and E.C. Prescott (1980). "Post-war U.S. Business Cycles," Carnegie Mellon University Working Paper.

Ionescu, V., C. Oara and M. Weiss (1997). "General Matrix Pencil Techniques for the Solution of Algebraic Riccati Equations: A Unified Approach." IEEE Transactions on Automatic Control, $42,8,1085-1097$.

Jenkins, G.M. and A.S. Alavi (1981). "Some Aspects of Modelling and Forecasting Multivariate Time Series," Journal of Time Series Analysis, 2, 1, 1-47.

Kohn, R and Ansley, C.F. (1986). "Estimation, Prediction, and Interpolation for ARMMA Models with Missing Data," Journal of the American Statistical Association, 81, 751-761.

Koopman, S.J., A.C. Harvey, J.A. Doornik and N. Shephard (1995). Stamp 5.0 Structural Time Series Analyser, Modeller and Predictor, Chapman \& Hall, London.

Petkov, P. Hr., N.D. Christov and M.M. Konstantinov (1991). Computational Methods for Linear Control Systems, Prentice-Hall, Englewood Cliffs, New Jersey.

Planas, C. (1997). Applied Time Series Analysis: Modeling, Forecasting, Unobserved Components Analysis and the Wiener-Kolmogorov Filter, Eurostat. (This work can be downloaded at http://europa.eu.int/en/comm/eurostat/research/noris4/)

Pole, A., M. West and J. Harrison (1994). Applied Bayesian Forecasting and Time Series Analysis, Chapman \& Hall, London. 
Shiskin, J., A.H. Young and J.C. Musgrave (1967). "The X-11 Variant of the Census Method Seasonal Adjustment Program," Technical Paper, Bureau of the Census (Washington D.C.)

Terceiro, J. (1990). Estimation of Dynamic Econometric Models with Errors in Variables. SpringerVerlag, Berlin.

West, M. (1997). "Time Series Decomposition and Analysis in a Study of Oxygen Isotope Records," Biometrika, 84, 489-494

Young, P.C. and J. Brenner (1991). MicroCAPTAIN Handbook: Version 2.0, Lancaster University: Centre for Research on Environmental Systems and Statistics, Lancaster, U.K.

Young, P., D.J. Pedregal and W. Tych (1999). "Dynamic Harmonic Regression," Journal of Forecasting, 18, 369-394.
Table 1. Correspondence between the state variables and the structural components.

\begin{tabular}{|l|l|l|l|}
\hline \multicolumn{2}{|l|}{$\operatorname{eig}(\bar{\Phi})$} & Spectral peaks at & Structural component \\
\hline Real & $\lambda_{j}=1$ & $f_{j}=0$ & Trend \\
\cline { 3 - 4 } & $\lambda_{j}=a_{j}-1 \leq a_{j}<1$ & $f_{j}=0$ if $0<a_{j}<1$ & Cycle \\
\cline { 3 - 4 } & & $\begin{array}{l}\text { If } a_{j}=0, \text { then } x_{j} \text { is a redundant state, which has no effect on } \\
\text { the structural components }\end{array}$ \\
\cline { 3 - 4 } & $f_{j}=1 / 2$ if $-1 \leq a_{j}<0$ & $\begin{array}{l}\text { Seasonal if } 1 / 2 \in F_{S}, \text { Cycle } \\
\text { otherwise }\end{array}$ \\
\hline Complex & $\lambda_{j, k}=a_{j} \pm b_{j} i$ & $f_{j, k}=(2 \pi)^{-1} \arctan \left(b_{j} / a_{j}\right)$ & $\begin{array}{l}\text { Seasonal if } f_{j, k} \in F_{S}, \text { Cycle } \\
\text { otherwise }\end{array}$ \\
\hline
\end{tabular}


Table 3. Structure of data generating process in SS form.

Table 2: Definition of the data generating process.

\begin{tabular}{|c|c|c|}
\hline Component & STSM representation & ARIMA representation \\
\hline Trend & $\begin{array}{l}T_{t+1}=T_{t}+\Delta_{t} \\
\Delta_{t+1}=\Delta_{t}+\eta_{t}\end{array}$ & $(1-B)^{2} T_{t+1}=\eta_{t}$ \\
\hline Seasonal & $\left(1+B+B^{2}+B^{3}\right) S_{t+1}=\omega_{t}$ & $\left(1+B+B^{2}+B^{3}\right) S_{t+1}=\omega_{t}$ \\
\hline Time series & $z_{t}=T_{t}+S_{1}+e_{t}$ & $\begin{array}{l}(1-B)\left(1-B^{4}\right) z_{t} \\
=\left(1-.933 B+.091 B^{2}-.047 B^{3}-.585 B^{4}+.548 B^{5}\right) a_{t} \\
\propto(1-.933 B)\left(1-.585 B^{4}\right) a_{,} ; a_{t} \sim \operatorname{nid}(0,1.824)\end{array}$ \\
\hline
\end{tabular}

\begin{tabular}{|c|c|c|c|c|c|c|c|c|c|}
\hline \multirow[b]{3}{*}{ Outputs } & \multicolumn{8}{|c|}{ Inputs } & \multirow{3}{*}{$\operatorname{eig}(\Phi)$} \\
\hline & $T_{t}$ & $\Delta_{t}$ & $S_{t}$ & $S_{t-1}$ & $S_{t-2}$ & $\eta_{t}$ & $\omega_{2}$ & $\varepsilon_{t}$ & \\
\hline & \multicolumn{5}{|c|}{$\Phi$} & \multicolumn{2}{|c|}{$\boldsymbol{E}$} & - & \\
\hline$T_{t+1}$ & 1 & 1 & 0 & 0 & 0 & 0 & 0 & - & 1 \\
\hline$\Delta_{t+1}$ & 0 & 1 & 0 & 0 & 0 & 1 & 0 & - & 1 \\
\hline$S_{t+1}$ & 0 & 0 & -1 & -1 & -1 & 0 & 1 & - & $+i$ \\
\hline$S_{t}$ & 0 & 0 & 1 & 0 & 0 & 0 & 0 & - & \\
\hline$S_{\mathrm{f}-3}$ & 0 & 0 & 0 & 1 & 0 & 0 & 0 & - & -1 \\
\hline \multirow[b]{2}{*}{$z_{t}$} & \multicolumn{5}{|c|}{$\boldsymbol{H}$} & \multicolumn{2}{|c|}{-} & C & \\
\hline & 1 & 0 & 1 & 0 & 0 & - & - & 1 & \\
\hline
\end{tabular}


Table 4. Structure of the data generating process in block-diagonal innovations form.

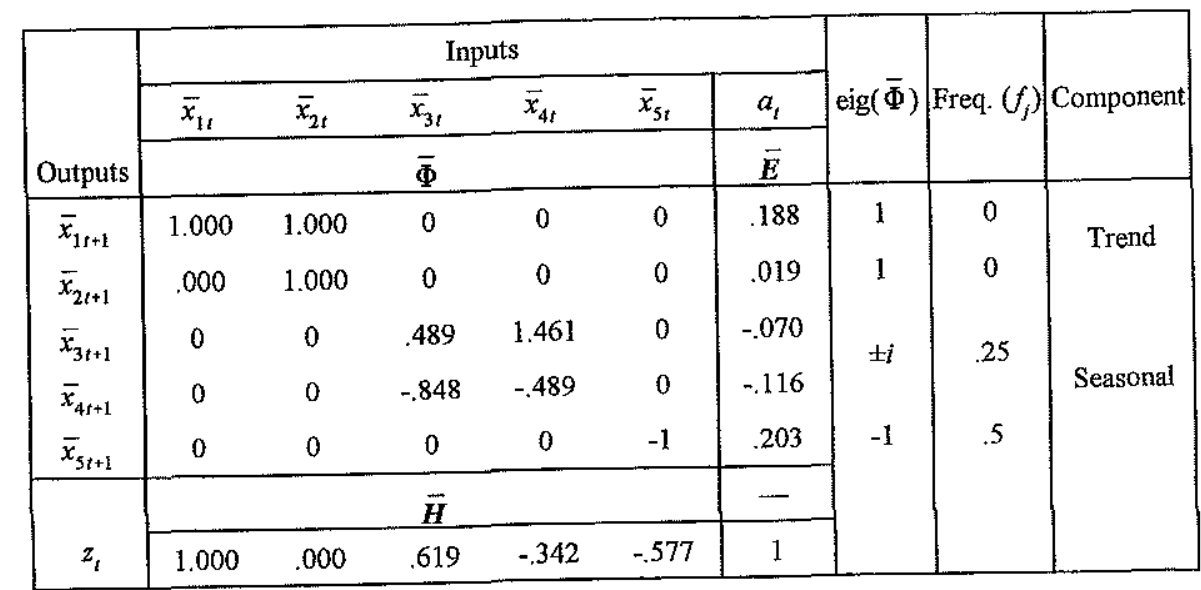

Table 5: Comparison between the models for the structural components in ARIMA notation.

\begin{tabular}{|c|c|c|}
\hline Component & Data generating process & Innovations model \\
\hline Trend & $\begin{array}{l}(1-B)^{2} T_{\mathrm{t}}=\eta_{t-1} \\
\eta_{t-1} \sim \operatorname{mid}(0,1 / 1600)\end{array}$ & $(1-B)^{2} t_{t}=(1-.899 B) \cdot 188 \varepsilon_{t-1}$ \\
\hline Seasonal & $\begin{array}{l}\left(1+B+B^{2}+B^{3}\right) S_{t}=\omega_{t-1} \\
\omega_{t-1} \sim \operatorname{nid}(0, .1)\end{array}$ & $\begin{array}{l}\left(1+B+B^{2}+B^{3}\right) s_{t} \\
\quad=\left(1+1.402 B+2.347 B^{2}\right)(-.120) \varepsilon_{t-1}\end{array}$ \\
\hline Irregular & $e_{t} \sim \operatorname{nid}(0,1)$ & $\varepsilon_{\mathrm{t}} \sim \operatorname{nid}(0,1.824)$ \\
\hline
\end{tabular}


Table 6. Structure of model (36) in block-diagonal innovations form

\begin{tabular}{|c|c|c|c|c|c|c|c|c|c|}
\hline \multirow[b]{3}{*}{ Outputs } & \multicolumn{7}{|c|}{ Inputs } & \multirow[b]{3}{*}{$\operatorname{eig}(\bar{\Phi})$} & \multirow[b]{3}{*}{ Component } \\
\hline & $\bar{x}_{1 t}$ & $\bar{x}_{2 t}$ & $\vec{x}_{3 t}$ & $\bar{x}_{4 t}$ & $u_{t}=1$ & $\hat{a}_{1 t}$ & $\hat{a}_{2 t}$ & & \\
\hline & \multicolumn{4}{|c|}{$\bar{\Phi}$} & $\bar{\Gamma}$ & \multicolumn{2}{|c|}{$\bar{E}$} & & \\
\hline $\bar{x}_{1 t+1}$ & 1 & 0 & 0 & 0 & 0 & .522. & .844 & 1 & Trend \\
\hline $\bar{x}_{2 t+1}$ & 0 & .231 & 0 & 0 & .026 & .231 & 0 & .231 & Cycle \\
\hline $\bar{x}_{3 t+1}$ & 0 & 0 & .333 & -.866 & 0 & & & \multirow[t]{5}{*}{$.013 \pm .496 i$} & \multirow{5}{*}{$\begin{array}{c}\text { Cycle } \\
\text { (harmonic) }\end{array}$} \\
\hline $\bar{x}_{4 t+1}$ & 0 & 0 & .403 & -.307 & 0 & -.140 & .103 & & \\
\hline \multirow{3}{*}{$z_{1 t}$} & \multicolumn{4}{|c|}{$\overline{\boldsymbol{H}}$} & $D$ & \multicolumn{2}{|c|}{-} & & \\
\hline & 0 & 1 & 0 & 0 & .110 & 1 & 0 & & \\
\hline & .971 & 0 & 1.106 & -.391 & 0 & 0 & 1 & & \\
\hline
\end{tabular}

Figure 1.a. Trace of $\boldsymbol{P}_{t \mid N}$ for models (26)-(27).
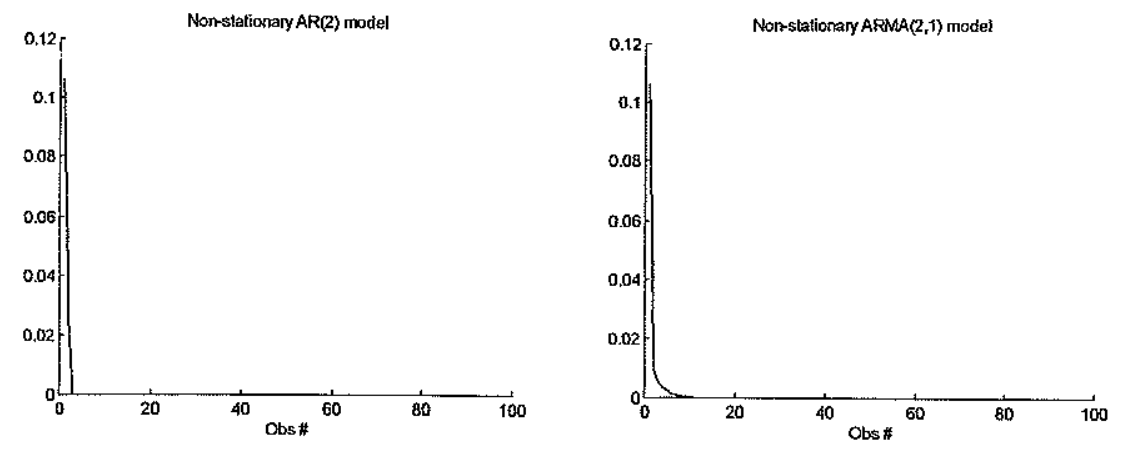

Figure 1,b. Trace of $P_{t \mid N}$ for model (28).
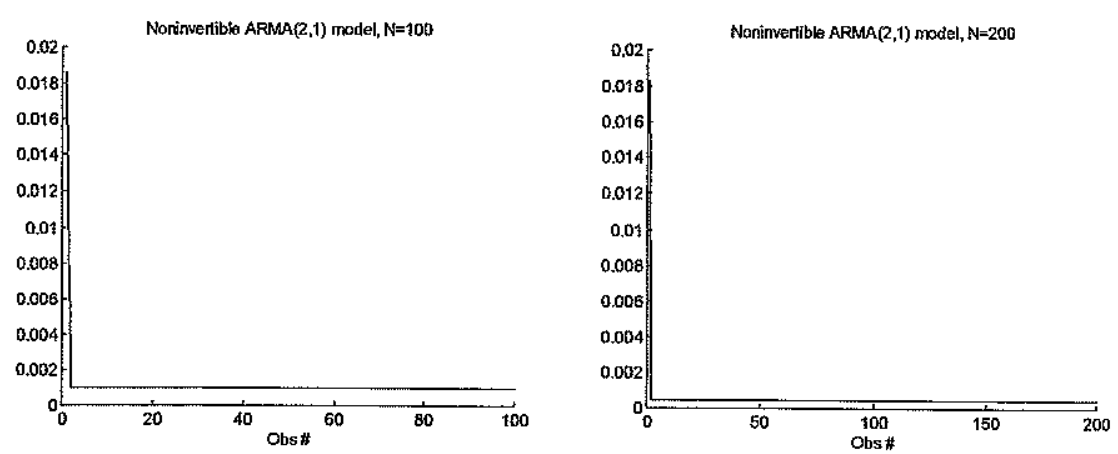
Figure 2. Comparison between the components obtained from the STSM and the innovations model.
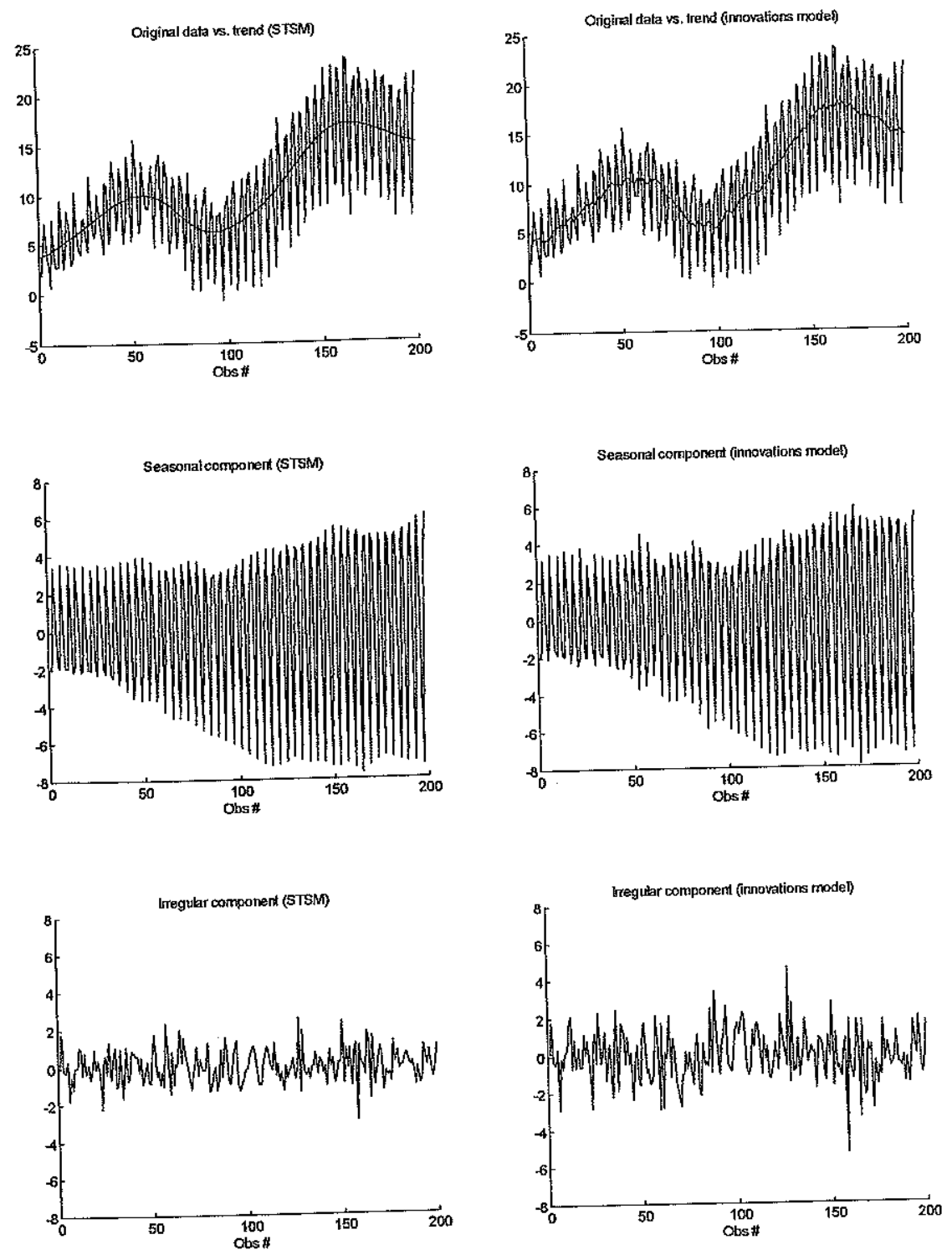

Figure 3 Comparison between the variances of the components implied by the STSM ("+") and the innovations model (continuous line)
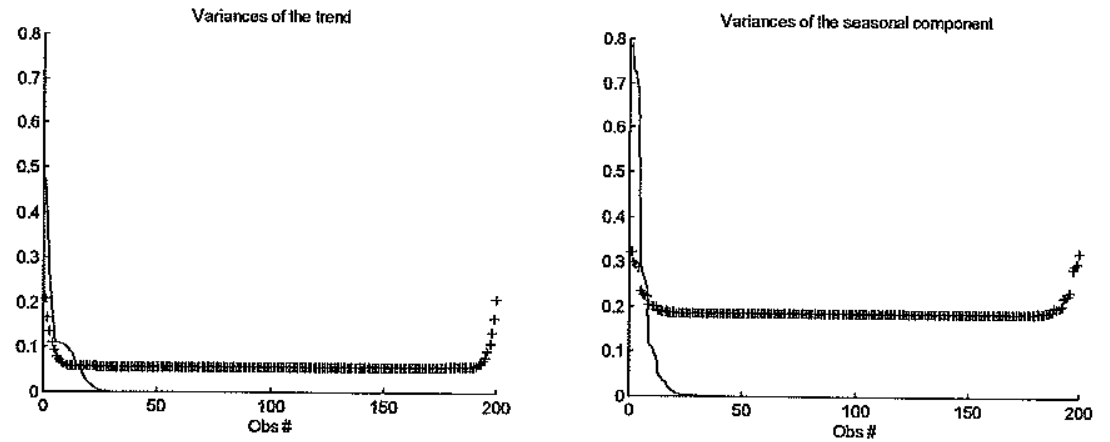
Figure 4. Autocorrelation analysis of the second-order differences of the trends implied by the STSM and the innovations model $†$.
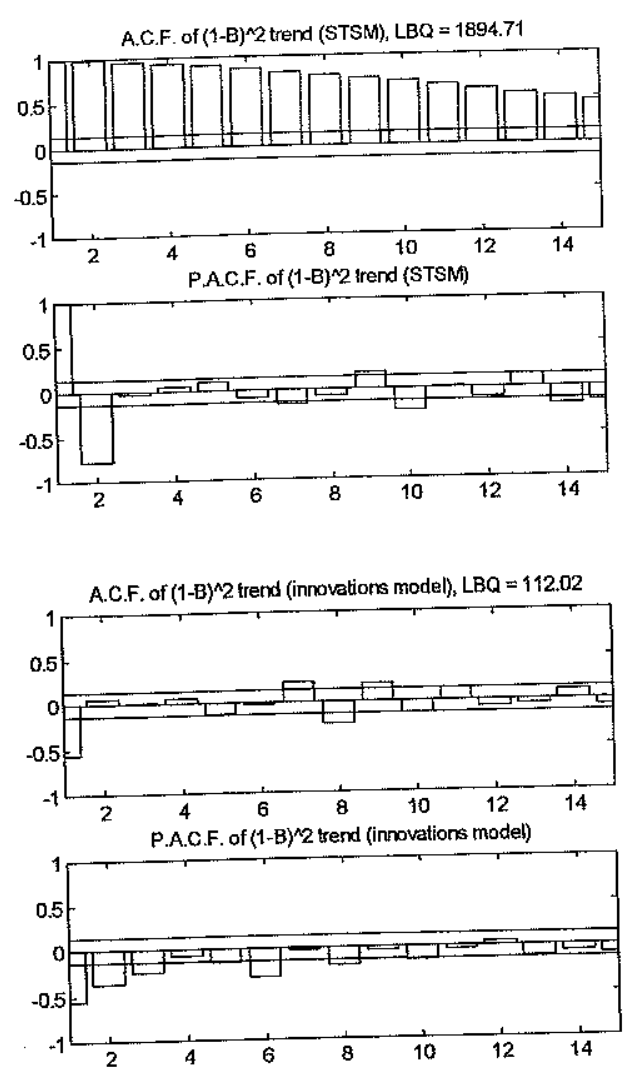

†Th value " $L B Q$ " appearing at the header of each plot is the Ljung-Box $Q$ statistic computed with the first 15 lags of the sample autocorrelation function.
Figure 5.a. Comparison between the derivatives of the trend implied by the STSM ("+") and the innovations model (continuous line).

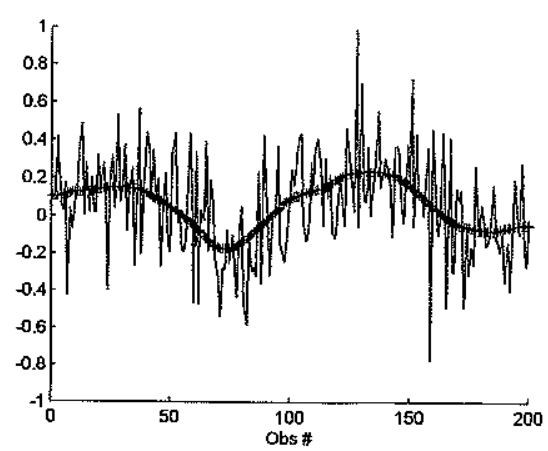

Figure 5.b Comparison between the derivatives of the trend after the ex-post smoothing.

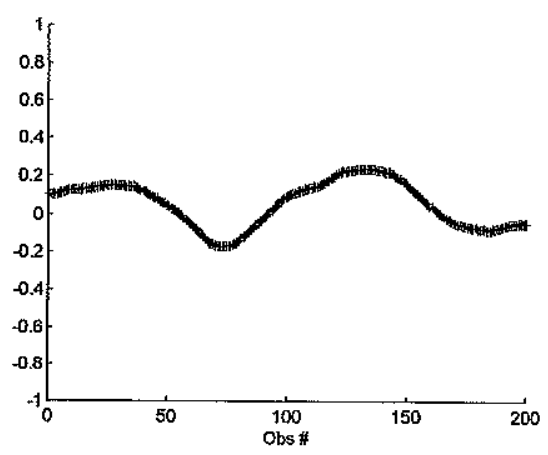


Figure 6. Trace of the FIS covariance of the states.

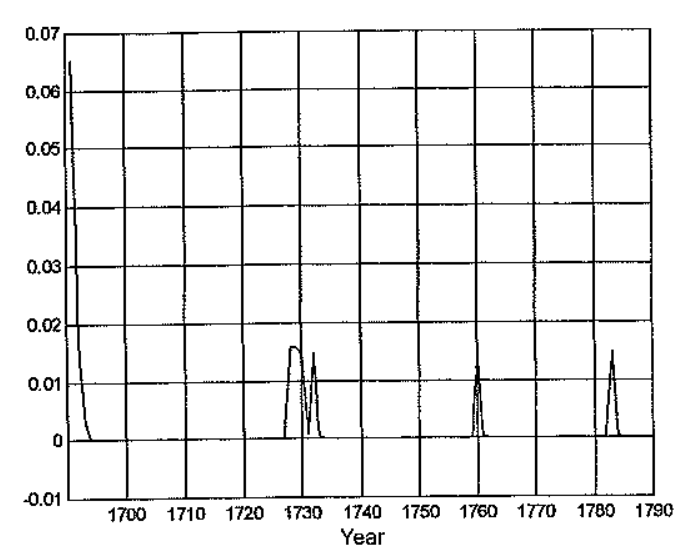

Figure 7. Common features of wheat prices (in logs)

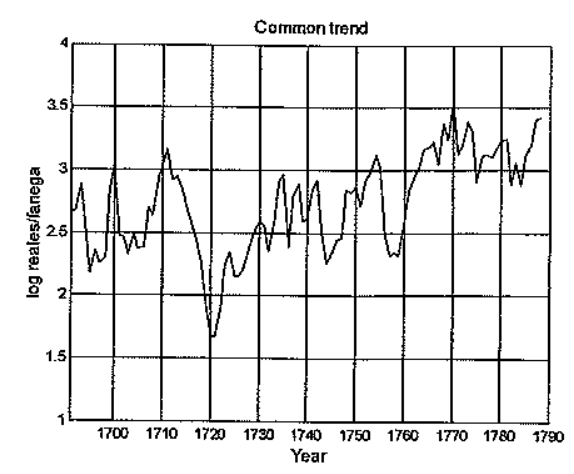

Common cyck

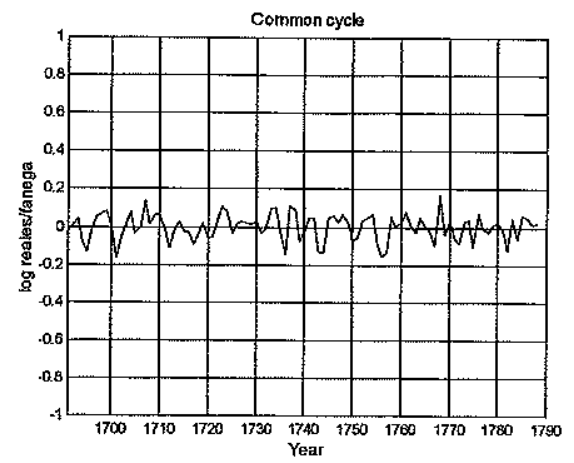


Appendix A: Simulated data used in Section 5.

\begin{tabular}{|c|c|c|c|c|c|c|c|c|c|}
\hline Obs. & $z_{t}$ & Obs. & $z_{f}$ & Obs. & $z_{1}$ & Obs. & $z_{t}$ & Obs. & $z_{t}$ \\
\hline 1 & 1.9839 & 41 & 6.5252 & 81 & 0.4915 & 121 & 1.5334 & 161 & 9.5232 \\
\hline 2 & 4.1365 & 42 & 7.8095 & 82 & 6.0958 & 122 & 8.5688 & 162 & 13.8117 \\
\hline 3 & 7.2564 & 43 & 12.7802 & 83 & 10.0224 & 123 & 13.5509 & 163 & 23.6179 \\
\hline 4 & 4.0816 & 44 & 11.6161 & 84 & 7.8136 & 124 & 14.4775 & 164 & 23.3728 \\
\hline 5 & 2.7511 & 45 & 5.1148 & 85 & 0.2390 & 125 & 2.4660 & 165 & 7.5703 \\
\hline 6 & 0.6828 & 46 & 7.4685 & 86 & 7.2630 & 126 & 9.1358 & 166 & 16.3808 \\
\hline 7 & 7.5726 & 47 & 13.8770 & 87 & 8.5484 & 127 & 17.6577 & 167 & 20.6114 \\
\hline 8 & 3.6843 & 48 & 10.4788 & 88 & 10.7379 & 128 & 13.3015 & 168 & 21.5621 \\
\hline 9 & 2.7747 & 49 & 5.2899 & 89 & 1.4390 & 129 & 6.2545 & 169 & 10.0252 \\
\hline 10 & 2.8758 & 50 & 9.1778 & 90 & 5.4900 & 130 & 9.6068 & 170 & 15.5322 \\
\hline 11 & 9.5626 & 51 & 15.5739 & 91 & 7.8018 & 131 & 15.4648 & 171 & 22.4384 \\
\hline 12 & 6.5804 & 52 & 10.9532 & 92 & 9.1682 & 132 & 15.8465 & 172 & 20.0040 \\
\hline 13 & 2.6921 & 53 & 6.1788 & 93 & 0.9772 & 133 & 4.3906 & 173 & 9.4084 \\
\hline 14 & 4.2375 & 54 & 7.3731 & 94 & 7.7581 & 134 & 10.6376 & 174 & 13.5416 \\
\hline 15 & 8.5775 & 55 & 13.3941 & 95 & 7.7703 & 135 & 16.9306 & 175 & 21.2057 \\
\hline 16 & 6.3674 & 56 & 10.6307 & 96 & 8.1144 & 136 & 18.0164 & 176 & 20.1215 \\
\hline 17 & 2.9625 & 57 & 8.6376 & 97 & -0.6658 & 137 & 5.5506 & 177 & 10.9987 \\
\hline 18 & 3.6781 & 58 & 8.6398 & 98 & 5.7581 & 138 & 11.8807 & 178 & 13.7982 \\
\hline 19 & 10.4649 & 59 & 11.7467 & 99 & 8.8322 & 139 & 18.1150 & 179 & 22.0740 \\
\hline 20 & 6.6928 & 60 & 12.9966 & 100 & 9.8072 & 140 & 17.9973 & 180 & 20.7452 \\
\hline 21 & 4.6681 & 61 & 4.6345 & 101 & 0.8696 & 141 & 6.9070 & 181 & 9.2546 \\
\hline 22 & 3.9816 & 62 & 8.7030 & 102 & 7.6816 & 142 & 11.0437 & 182 & 13.3336 \\
\hline 23 & 7.6172 & 63 & 13.0195 & 103 & 10.5368 & 143 & 19.6207 & 183 & 21.2908 \\
\hline 24 & 7.6901 & 64 & 14.1113 & 104 & 11.3036 & 144 & 19.4881 & 184 & 21.5541 \\
\hline 25 & 5.5549 & 65 & 5.1189 & 105 & 0.2456 & 145 & 8.2034 & 185 & 9.0118 \\
\hline 26 & 4.3479 & 66 & 9.7714 & 106 & 6.1984 & 146 & 12.0909 & 186 & 13.0161 \\
\hline 27 & 12.0189 & 67 & 13,4173 & 107 & 8.9129 & 147 & 18.7055 & 187 & 20.4928 \\
\hline 28 & 7.4748 & 68 & 11.7304 & 108 & 11.5264 & 148 & 20.7599 & 188 & 20.4763 \\
\hline 29 & 5.7602 & 69 & 4.0712 & 109 & 1.1415 & 149 & 8.5824 & 189 & 7.3662 \\
\hline 30 & 6.4299 & 70 & 6.3484 & 110 & 8.7756 & 150 & 16.3149 & 190 & 12.6438 \\
\hline 31 & 10.0989 & 71 & 11.7760 & 111 & 11.4379 & 151 & 18.8405 & 191 & 18.7619 \\
\hline 32 & 8.1723 & 72 & 10.8252 & 112 & 12.1508 & 152 & 22.4995 & 192 & 20.0436 \\
\hline 33 & 6.8097 & 73 & 4.6275 & 113 & 0.6590 & 153 & 8.8153 & 193 & 9.2562 \\
\hline 34 & 4.0742 & 74 & 6.5335 & 114 & 9.5043 & 154 & 15.1631 & 194 & 12.8691 \\
\hline 35 & 11.2922 & 75 & 11.9646 & 115 & 10.7862 & 155 & 19.4532 & 195 & 19.3712 \\
\hline 36 & 11.0120 & 76 & 9.8638 & 116 & 12.8343 & 156 & 22.7587 & 196 & 21.7131 \\
\hline 37 & 5.0782 & 77 & 2.3814 & 117 & 0.5977 & 157 & 10.7683 & 197 & 7.4288 \\
\hline 38 & 6.0249 & 78 & 6.6286 & 118 & 8.2299 & 158 & 11.0380 & 198 & 11.4007 \\
\hline 39 & 13.4450 & 79 & 12.2037 & 119 & 10.9629 & 159 & 22.5336 & 199 & 19.2632 \\
\hline 40 & 113456 & 80 & 80208 & 120 & 13.4723 & 160 & 22.4050 & 200 & 21.9119 \\
\hline
\end{tabular}

13.4723

$160 \quad 22.4050$

$200 \quad 21.9119$
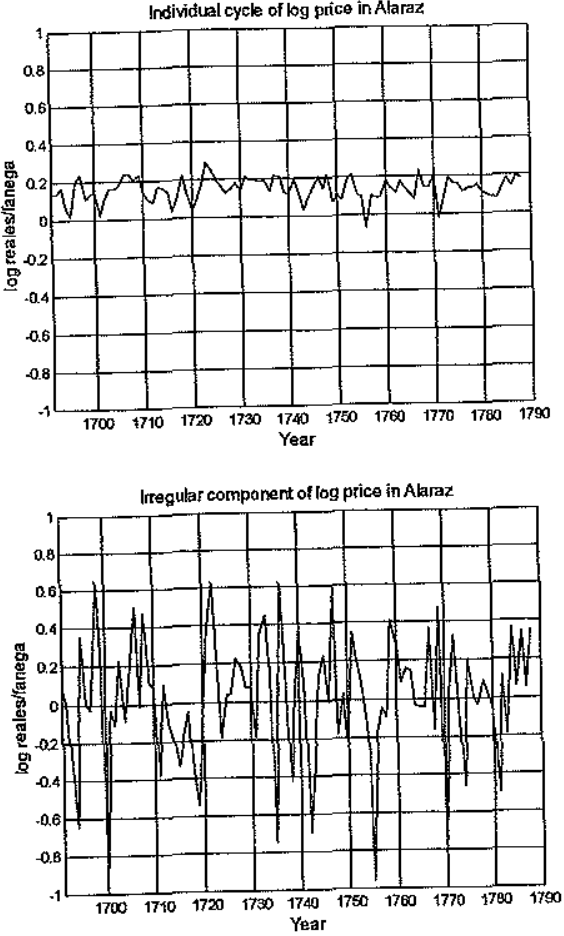

Iregular component of $\log$ price in Sandoval

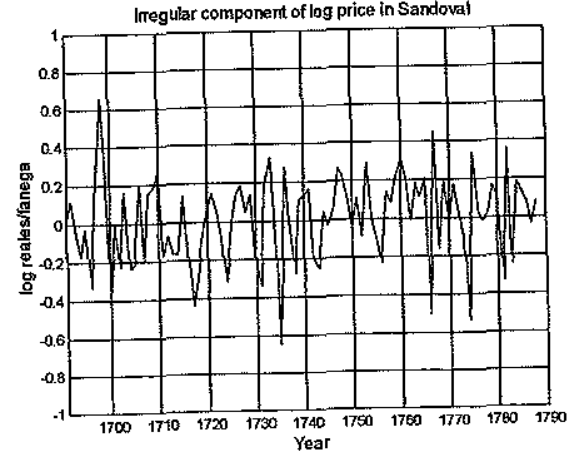


Appendix B: Average price (in reales per fanega) of the wheat sold in the Monasterio de Sandoval $\left(S_{t}\right)$ and the Fábrica de la iglesia de Alaraz $\left(A_{t}\right) \dagger$.

\begin{tabular}{rrrrrrr}
\hline Year & $S_{t}$ & $A_{t}$ & & Year & $S_{t}$ & $A_{t}$ \\
\cline { 6 - 8 } 1691 & 14.88 & 16.33 & & 1727 & 11.56 & NA \\
1692 & 17.67 & 22.32 & & 1728 & 11.86 & NA \\
1693 & 15.38 & 12.69 & & 1729 & 14.33 & NA \\
1694 & 10.17 & 6.76 & & 1730 & 10.81 & 17.00 \\
1695 & 7.50 & 11.26 & & 1731 & 8.87 & NA \\
1696 & 7.55 & 13.00 & & 1732 & 12.42 & 18.00 \\
1697 & 12.33 & 12.30 & & 1733 & 20.42 & 28.00 \\
1698 & 20.81 & 23.00 & & 1734 & 19.06 & 28.00 \\
1699 & 23.63 & 28.00 & & 1735 & 10.00 & 11.00 \\
1700 & 14.66 & 10.00 & & 1736 & 12.37 & 20.00 \\
1701 & 10.00 & 10.00 & & 1737 & 18.00 & 28.00 \\
1702 & 8.75 & 11.00 & & 1738 & 15.00 & 16.00 \\
1703 & 12.10 & 15.18 & & 1739 & 13.83 & 14.00 \\
1704 & 10.37 & 14.08 & & 1740 & 15.25 & 22.00 \\
1705 & 8.39 & 14.52 & & 1741 & 21.40 & 23.00 \\
1706 & 13.22 & 22.92 & & 1742 & 15.81 & 11.00 \\
1707 & 14.08 & 21.37 & & 1743 & 8.25 & 8.00 \\
1708 & 16.50 & 28.00 & & 1744 & 8.83 & 10.58 \\
1709 & 24.10 & 28.00 & & 1745 & 10.55 & 16.00 \\
1710 & 29.00 & 28.00 & & 1746 & 13.00 & 15.00 \\
1711 & 20.00 & 18.00 & & 1747 & 15.68 & 25.00 \\
1712 & 15.45 & 20.00 & & 1748 & 23.04 & 19.00 \\
1713 & 15.95 & 20.00 & & 1749 & 18.91 & 19.00 \\
1714 & 14.90 & 17.00 & & 1750 & 15.84 & 14.00 \\
1715 & 16.50 & 12.00 & & 1751 & 15.96 & 22.00 \\
1716 & 10.87 & 11.00 & & 1752 & 17.70 & 28.00 \\
1717 & 6.66 & 11.00 & & 1753 & 28.00 & 28.00 \\
1718 & 6.96 & 8.00 & & 1754 & 23.50 & 22.40 \\
1719 & 6.75 & 5.00 & & 1755 & 17.16 & 8.00 \\
1720 & 5.43 & 5.00 & & 1756 & 8.25 & 8.00 \\
1721 & 5.85 & 8.00 & & 1757 & 10.25 & 9.50 \\
1722 & 7.40 & 16.00 & & 1758 & 12.00 & 11.00 \\
1723 & 9.69 & 18.00 & & 1759 & 12.62 & NA \\
1724 & 8.25 & 12.00 & & 1760 & 18.93 & 22.00 \\
1725 & 8.25 & 10.50 & & 1761 & 23.00 & 23.50 \\
1726 & 10.12 & 10.89 & & 1762 & 19.16 & 25.50
\end{tabular}

\begin{tabular}{rrr}
\hline Year & $S_{t}$ & $A_{\mathrm{t}}$ \\
\hline 1763 & 24.14 & 28.00 \\
1764 & 28.00 & 28.00 \\
1765 & 29.87 & 26.00 \\
1766 & 15.00 & 25.56 \\
1767 & 30.10 & 35.00 \\
1768 & 29.30 & 34.00 \\
1769 & 30.00 & 46.00 \\
1770 & 35.25 & 19.92 \\
1771 & 25.75 & 22.14 \\
1772 & 23.58 & 33.92 \\
1773 & 27.58 & 34.00 \\
1774 & 16.75 & 21.00 \\
1775 & 23.25 & 23.58 \\
1776 & 25.00 & 27.41 \\
1777 & 22.50 & 25.09 \\
1778 & 22.50 & 27.30 \\
1779 & 28.37 & 28.70 \\
1780 & 29.25 & 27.91 \\
1781 & 18.00 & 16.76 \\
1782 & 22.75 & NA \\
1783 & 17.37 & 19.78 \\
1784 & 20.25 & 28.00 \\
1785 & 28.00 & 31.18 \\
1786 & 28.00 & 41.87 \\
1787 & 29.37 & 39.42 \\
1788 & 33.75 & 52.75
\end{tabular}

$\dagger$ NA: Not available. 